\title{
Medical staff perceptions of risk communication needs for the public and comparison with the needs expressed by the public
}

\author{
Y. Yoshida ${ }^{1, *}$ and Y. Yoshida ${ }^{2}$ \\ ${ }^{1}$ Faculty of Medical Sciences, Shubun University, 6 Nikko-cho, Ichinomiya, Japan. \\ ${ }^{2}$ Graduate School of Pharmaceutical Sciences, Nagoya City University, 3-1 Tanabe-dori, Mizuho-ku, Nagoya 467-8603, Japan.
}

Received: 11 December 2019 / Accepted: 6 July 2020

\begin{abstract}
Risk communication programs about radiation exposure should be conducted continuously and rigorously by healthcare workers in the Tohoku region in order to reduce the stress caused by radiation exposure. This study aimed to compare the perception by medical staff of the public need to the public need as defined in a previous study, as well as examine the level of perception among healthcare workers of the two concepts "as low as reasonably achievable" (ALARA), and Regulatory Sciences. These two concepts were expected to be the ones to impart information regarding the effect of radiation on the human body during risk communication. The results showed differences between the public perception of risks and belief of what the public risk perception was by the medical staff. In addition, only $23.5 \%$ and $16.5 \%$ medical doctors had accurate perception of ALARA and Regulatory Sciences in Japan, respectively, even after the great East Japan Earthquake., ALARA and Regulatory Sciences should be added to healthcare workers' education curriculums to enhance their knowledge level of these concepts. From the viewpoint of laypersons, public health awareness programs conducted by the local and central governments ranked fourth and fifth respectively as sources of information. One of the reasons was that, to some extent, the attitude of the central government seemed apt to be overbearing to laypersons. Therefore, we also believe that medical doctors are expected to be intermediaries between central/local governments and laypersons.
\end{abstract}

Keywords: as low as reasonably achievable (ALARA) / regulatory sciences / risk communication / perception gap / the great East Japan Earthquake

\section{Introduction}

After the great East Japan Earthquake hit the Tohoku region on March 11, 2011, risk communication activities have been organized and conducted by various stakeholders, especially in the affected region, to reduce the anxiety and stress of the evacuees with respect to radiation exposure (Sekizawa, 2013; Yamaguchi, 2013; Kuroda et al., 2018). In order to increase the efficacy of these activities, risk communication activities in the affected area have been reported in several studies (Staudenherz and Leitha, 2011; Bromet, 2014; Nakashima et al., 2015; Goto et al., 2015; Ohtsuru et al., 2015; Yumiya et al., 2019).

In order to perform risk communication activities in the affected area smoothly and effectively, the efficacy of risk communications should be evaluated. Therefore, we have also conducted a previous study to evaluate the effectiveness of risk communication with respect to the recognition level of technical terms relevant to radiation exposure to the human body among residents of the six prefectures of Miyagi,

*Corresponding author: yoshida.yo@subun.ac.jp
Fukushima, Tokyo, Aichi, Hiroshima, and Nagasaki in Japan. Miyagi and Fukushima were selected as affected areas following the great East Japan Earthquake. Tokyo and Aichi were selected as controls. Hiroshima and Nagasaki were selected as the atom-bombed areas. The study showed that, based on the differences among the six prefectures on the recognition level of the 20 technical terms regarding radiation, respondents in Fukushima showed the highest interest in radiation-relevant issues, followed by those in Miyagi (Yoshida et al., 2017). Our previous research activities have also shown that when the concept of Regulatory Science and ALARA were appropriately communicated to the audience, risk communication was conducted more smoothly.

Healthcare workers played important roles in risk communication as lecturers and/or counselors. Our research demonstrated gaps in perception of pharmaceutical terms and related issues between laypeople and medical doctors (Yoshida et al., 2013), pharmacists (Yoshida and Yoshida, 2014), and nurses (Yoshida and Yoshida, 2015). Communication between healthcare providers and patients had been limited by overestimation by providers of patients' recognition level of medical terms. The gaps between the basic recognition of medical terms by laypeople and the same as estimated by the 
medical doctors suggested that the possibility should be considered that patients were not able to recognize much more difficult terms. Compared to medical doctors, pharmacists tended to overestimate patients' recognition level of medical terms. Differences between medical doctors and nurses with respect to the estimation of patients' level of medical term recognition were also assessed. The level of medical term recognition by patients was higher when estimated by nurses than when estimated by medical doctors. Lochard et al. mentioned the importance of the dialogue meetings initiated by ICRP at Fukushima, and that these dialogues were centered on the public interest, and not on what the medical staff believed about the public interest. (Lochard et al., 2019). Therefore, the present research compared the perception by medical staff of the public need to the public need as defined in a previous study.

The concept of Regulatory Sciences was proposed by Dr. Mitsuru Uchiyama in 1987 to harmonize scientific and technological achievements of people and society and make them truly useful. Currently, this concept of establishing standard values based on the latest scientific and technological achievements has spread as a basic idea from the perspective of how much risk can be accepted within safety regulations in risk-related fields (Hayashi, 2009; Poston and Fortieth Lauriston, 2017). The meaning of ALARA is defined as follows; "the principle of optimization of protection: the likelihood of incurring exposures, the number of people exposed, and the magnitude of their individual doses should all be kept as low as reasonably achievable, taking into account economic and societal factors" (ICRP Publication 103, 2007). In Japan, some researchers, considering economic and social factors, have expressed concern about the effectiveness of the ALARA concept (Tsuchida, 2018). Based on the benefit of learning from the experiences of the Fukushima accident, the International Radiation Protection Association published that: "It is universally accepted that the optimization principle is the central pillar for the practical implementation of radiation protection and is the dominant factor controlling exposures in any well-developed system of protection. The most widespread concern was in the interpretation of what is "reasonable" and proportionate, and that an overly simplistic approach is leading to continuing expectations of lower doses. There was a strong desire to identify methods of placing more formal recognition that ALARA does not generally mean continually lower doses, and that there is a balanced approach to optimization which results in value to society (Coates and Czarwinski, 2018)".

Therefore, this study also examined the level of perception of the two concepts of ALARA and Regulatory Sciences among healthcare workers.

\section{Materials and methods}

The study protocol was approved on July 14, 2016 by the Ethics Committee of Shubun University (approval number: 28SR2) before data collection. Demographic data of medical doctors, pharmacists, and nurses were collected in this study from all over Japan. Data of laypersons were collected in our previous study (Yoshida et al., 2017). We had previously assessed the perception gaps regarding technical terms of the effect of radiation on the human body and related issues among residents of the six Japanese prefectures of Miyagi, Fukushima, Tokyo, Aichi, Hiroshima, and Nagasaki.

Medical doctors, pharmacists, and nurses were examined as subjects in the current study. The research was performed under contract with NTT Rezonanto Co., Ltd. using an internet survey that was monitored by Goo Research contractors. The monitors were previously registered with an internet research company. Basic information on sex, residence, etc., of the monitors was registered as well. 170 medical doctors, 84 pharmacists, and 246 nurses were selected from all over Japan. We used a two-step selection method. Samples representing $110 \%$ of the target number were collected and submitted to us, after excluding imperfect samples. The research period for medical doctors, pharmacists, and nurses was August 12 to 17, 2016. The research period for the laypersons was February 13 to 19, 2014. As an incentive, each monitor got 50 points redeemable on the Goo website (worth about 50 cents) after completing the answers to the questions.

The scoring of the answers was calibrated on a scale of 1-5, one of which was selected as the most appropriate number by the respondents. For example, for perception of the concept of ALARA among medical doctors, pharmacists, nurses and laypersons, they chose answers calibrated on a scale of 1-5, wherein 1 indicated "I do not know at all", 3 indicated "I cannot tell clearly whether I know", and 5 indicated "I know very well". With respect to the perception of laypersons' recognition level of the 20 medical terms related to radiation and eight technical issues regarding radiation among medical doctors, pharmacists, and nurses, they chose answers calibrated on a scale of 1-5, wherein 1 indicated "I do not think that the layperson knows", 3 indicated "I cannot tell clearly whether the layperson knows or not", and 5 indicated "I think that the layperson knows". This recognition was termed as "laypersons' perception level of technical terms regarding the effect of radiation on the human body as estimated by healthcare workers of Japan". The most appropriate number between 1 and 5 was selected.

A Chi-square test and a binary logistic regression test were applied to analyze the data. In terms of applying the Chi-square test, scores of 4 and 5 were believed to indicate positive perception of "they know". Generally, when using the 5-point Likert scale, to make definition of the numbers clear, 1, 3 and 5 were explained explicitly, but 2 and 4 were explained implicitly. In terms of applying the binary logistic regression test to assess the crude odds ratio (OR) and adjusted OR with respect to the perception of laypersons' recognition level of the 20 medical terms related to radiation and eight technical issues regarding radiation among medical doctors, pharmacists, and nurses, the 5-point Likert scores of 20 terms were summed, and 61 was adopted as a cut-off index, so that a total score $\geq 61$ for recognition level of the 20 technical terms regarding radiation indicated that "laypersons know".

\section{Results}

Table 1 shows the demographic characteristics of the respondents. Between male and female medical doctors, a statistically significant difference was observed in age and participation in clinical trials. A statistically significant 


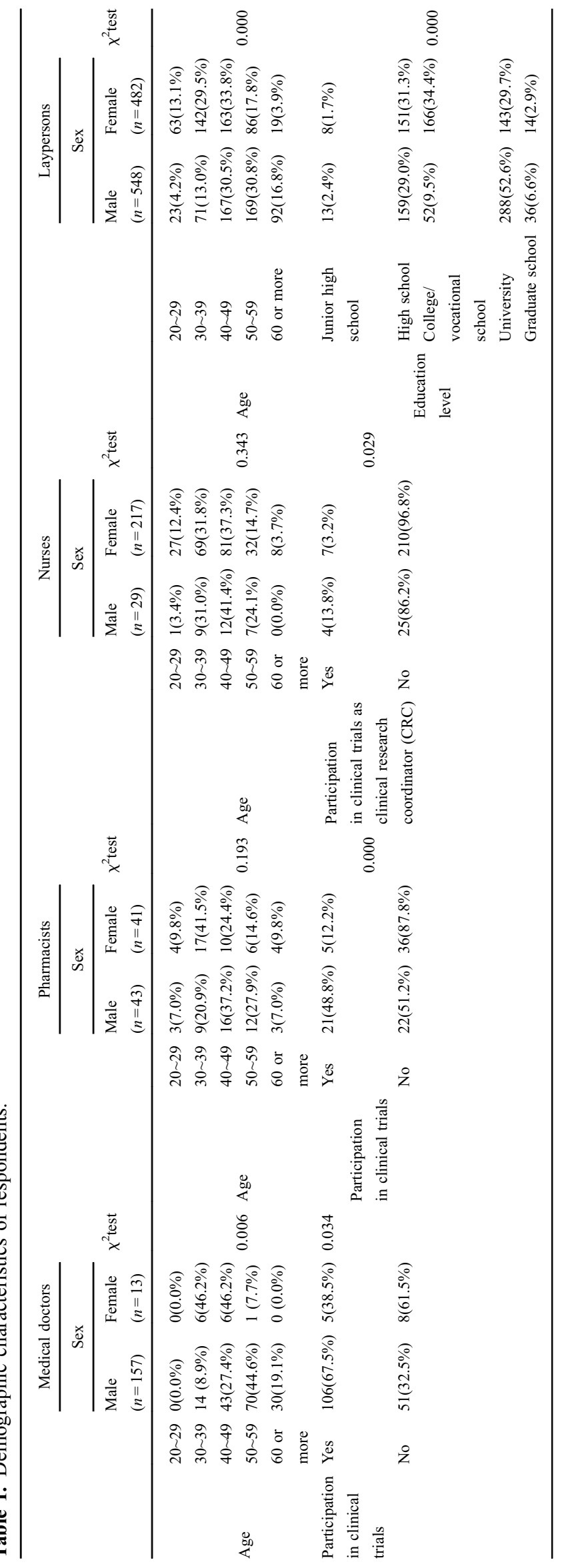


Table 2. Difference in the percentage of "knowing" concepts of ALARA and Regulatory Science among medical doctors, pharmacists and nurses.

\begin{tabular}{|c|c|c|c|c|}
\hline \multirow[b]{2}{*}{ Concept } & \multirow{2}{*}{$\frac{\text { Medical doctors }(n=170)}{\text { Know }(\%)}$} & \multirow{2}{*}{$\frac{\text { Pharmacists }(n=84)}{\text { Know }(\%)}$} & \multirow{2}{*}{$\frac{\text { Nurses }(n=246)}{\text { Know }(\%)}$} & \multirow[b]{2}{*}{$\chi^{2}$ tes } \\
\hline & & & & \\
\hline $\begin{array}{l}\text { Perception of the concept of "as low as } \\
\text { reasonably achievable" (ALARA) }\end{array}$ & 23.5 & 16.7 & 8.9 & 0.000 \\
\hline $\begin{array}{l}\text { Perception of the concept of Regulatory } \\
\text { Sciences }\end{array}$ & 16.5 & 17.9 & 7.3 & 0.004 \\
\hline $\begin{array}{l}\text { The standards regarding radiation based } \\
\text { on the concept of Regulatory Sciences }\end{array}$ & 16.5 & 15.5 & 8.1 & 0.023 \\
\hline
\end{tabular}

"According to percentage of scores 4 and $5 "$.

Table 3. Difference in the percentage of "Agree" on the reliability of central government and risk avoidance behavior regarding radiation among medical doctors, pharmacists, nurses and laypersons.

\begin{tabular}{|c|c|c|c|c|c|}
\hline & Medical doctors $(n=170)$ & Pharmacists $(n=84)$ & Nurses $(n=246)$ & Laypersons $(n=1030)$ & \\
\hline $\begin{array}{l}\text { Taking into account the uncertainty } \\
\text { of the risk assessment, laypersons } \\
\text { should avoid the risk at your own } \\
\text { risk }\end{array}$ & 32.4 & 29.8 & 33.3 & 54.0 & 0.000 \\
\hline
\end{tabular}

"According to percentage of scores 4 and 5".

difference was observed in participation in clinical trials between male and female pharmacists, as well as between male and female nurses. A statistically significant difference was also observed in age and education between male and female laypersons.

Table 2 showed that there were statistically significant differences in the percentage of "knowing" of the concept of ALARA and Regulatory Sciences among medical doctors, pharmacists, and nurses. The highest percentages of all three sources were marked by medical doctors, followed by pharmacists, and then nurses. $23.5 \%$ of medical doctors, $16.7 \%$ of pharmacists, and $8.9 \%$ of nurses had perception of the concept of ALARA. $16.5 \%$ of medical doctors, $17.9 \%$ of pharmacists, and $7.3 \%$ of nurses had perception of the concept of Regulatory Sciences. In addition, $16.5 \%$ of medical doctors, $15.5 \%$ of pharmacists, and $8.1 \%$ of nurses knew that the standards regarding radiation were set based on the concept of Regulatory Sciences.

As shown in Table 3, there was a statistically significant difference in the risk avoidance behavior regarding radiation. In terms of "taking into account the uncertainty of the risk assessment, laypersons should avoid the risk", the highest percentages $(54.0 \%)$ were marked by laypersons. From viewpoint of personal responsibility for avoiding risk, there was clearly mistrust of government by laypersons. However, there was no statistically significant difference in the reliability attributed to the central government. About one-fifth of each respondent group agreed that "by performing risk avoidance behavior according to the criteria defined by the government, laypersons can sufficiently avoid health risks". In terms of performing risk avoidance behavior, there was clearly mistrust of government recommendations both within medical staff and laypersons.

As shown in Table 4, the differences between six items of knowledge out of 10 that were delivered via a forum-style risk communication program were significant. For example, there was a statistically significant difference among medical doctors, pharmacists, nurses, and laypersons with respect to the 3 to 5 highest recognition levels of 10 terms such as "scientific facts", "effectiveness of food standards as a radiation countermeasure", and "setting the basis for the standards". The knowledge of "scientific facts" was marked by $80.6 \%, 77.4 \%, 72.4 \%$ and $60.2 \%$ of medical doctors, pharmacists, laypersons and nurses, respectively. The knowledge of "effectiveness of food standards as a radiation countermeasure" was marked by $73.8 \%, 73.3 \%, 67.6 \%$ and $61.0 \%$ of pharmacists, laypersons, medical doctors and nurses, respectively. The knowledge of "setting the basis for the standards" was marked by $71.8 \%, 70.2 \%, 69.3 \%$ and $55.7 \%$ of medical doctors, pharmacists, laypersons and nurses, respectively. On the other hand, there was also a statistically significant difference for the lowest recognition levels of 
Table 4. Difference in the percentage of "Need" of knowledge which should be delivered at the forum-style risk communication among medical doctors, pharmacists, nurses and laypersons.

\begin{tabular}{|c|c|c|c|c|c|}
\hline & Medical doctors $(n=170)$ & Pharmacists $(n=84)$ & Nurses $(n=246)$ & Laypersons $(n=1030)$ & \\
\hline Risk of radiation effects & 71.8 & 73.8 & 68.7 & 74.7 & 0.278 \\
\hline Radiation effects on children & 75.9 & 75.0 & 68.3 & 71.6 & 0.343 \\
\hline Scientific facts & 80.6 & 77.4 & 60.2 & 72.4 & 0.000 \\
\hline $\begin{array}{l}\text { Effectiveness of food standards } \\
\text { as a radiation countermeasure }\end{array}$ & 67.6 & 73.8 & 61.0 & 73.3 & 0.001 \\
\hline $\begin{array}{l}\text { Effectiveness of decontamination } \\
\text { as a radiation countermeasure }\end{array}$ & 66.5 & 66.7 & 63.8 & 68.3 & 0.606 \\
\hline Validity of the standards & 70.0 & 72.6 & 54.5 & 68.1 & 0.000 \\
\hline Recommendation of avoidance behavior & 65.9 & 66.7 & 58.5 & 65.0 & 0.242 \\
\hline $\begin{array}{l}\text { The effectiveness of the standards } \\
\text { of space dose of radiation as a } \\
\text { countermeasure }\end{array}$ & 60.0 & 64.3 & 54.1 & 66.4 & 0.003 \\
\hline
\end{tabular}

"According to percentage of scores 4 and 5".

Table 5. Difference in the percentage of "Agree" on the sources of information regarding radiation and its reliability among medical doctors, pharmacists, nurses and laypersons.

\begin{tabular}{|c|c|c|c|c|c|}
\hline & Medical doctors $(n=170)$ & Pharmacists $(n=84)$ & Nurses $(n=246)$ & Laypersons $(n=1030)$ & \\
\hline TV & 32.9 & 46.4 & 51.2 & 57.5 & 0.000 \\
\hline Newspaper & 37.1 & 39.3 & 43.1 & 52.8 & 0.000 \\
\hline Public relations by the local government & 22.4 & 27.4 & 33.3 & 37.9 & 0.000 \\
\hline Public relations by central government & 26.8 & 28.6 & 35.0 & 33.7 & 0.198 \\
\hline Radio & 16.5 & 15.5 & 26.8 & 25.9 & 0.009 \\
\hline Reliability & Agree $(\%)$ & Agree $(\%)$ & Agree $(\%)$ & Agree $(\%)$ & $\chi^{2}$ test \\
\hline Academic journals/ technical book & 75.9 & 66.7 & 53.3 & 51.2 & 0.000 \\
\hline Forum/Meeting of risk communication & 50.6 & 58.3 & 51.6 & 42.0 & 0.001 \\
\hline Internet & 20.0 & 23.8 & 22.0 & 29.3 & 0.014 \\
\hline Radio & 16.5 & 16.7 & 21.1 & 26.2 & 0.009 \\
\hline
\end{tabular}

"According to percentage of scores 4 and 5".

"uncertainty of radiation effects". It was marked by $70.0 \%$, $65.5 \%, 59.5 \%$ and $50.9 \%$ of medical doctors, pharmacists, laypersons and nurses, respectively.

Table 5 demonstrated statistically significant differences among respondents for seven out of the eight sources of information regarding radiation. $54.7 \%$ of medical doctors considered academic journals/technical book to be a resource, followed by the internet $(44.1 \%)$, forum/meeting of risk communication $(40.0 \%)$, with radio ranking the lowest
(16.5\%). $75.9 \%$ of medical doctors believed that academic journals/technical books were reliable, followed by forum/ meeting of risk communication $(50.6 \%)$, public relations by central government $(34.7 \%)$, with radio again ranking the lowest $(16.5 \%) .46 .4 \%$ of pharmacists considered TV to be a resource, followed by the internet $(42.9 \%)$, forum/meeting of risk communication $(41.7 \%)$, with radio again ranking the lowest $(15.5 \%) .66 .7 \%$ of pharmacists believed that academic journals/technical books were reliable, followed by forum/ 
Table 6. Odds ratios (OR) and 95\% confidence intervals (CI) for difference among medical doctors, pharmacists and nurses with respect to laypersons' expected recognition level of the 20 medical terms regarding radiation.

\begin{tabular}{|c|c|c|c|c|c|c|c|}
\hline & & $\begin{array}{l}\text { Know }^{a} \\
\text { N (\%) }\end{array}$ & $\begin{array}{l}\text { Crude OR } \\
(95 \% \mathrm{CI})^{\mathrm{b}}\end{array}$ & $p$ & & Adjusted OR $(95 \% \mathrm{CI})^{\mathrm{c}}$ & $p$ \\
\hline Sex & Female & $43 / 271$ & $(15.9)$ & 1 (Reference) & & 1 (Reference) & \\
\hline \multirow[b]{3}{*}{ Age } & $20-29$ & $7 / 35$ & $(20.0)$ & 1 (Reference) & & 1 (Reference) & \\
\hline & $30-39$ & $20 / 124$ & (16.1) & $0.769(0.296-2.002)$ & 0.591 & $0.653(0.248-1.719)$ & 0.388 \\
\hline & $40-49$ & $32 / 168$ & $(19.0)$ & $0.941(0.378-2.346)$ & 0.896 & $0.720(0.281-1.843)$ & 0.493 \\
\hline \multirow{3}{*}{ Occupation } & Nurse & $37 / 246$ & $(15.0)$ & 1 (Reference) & & 1 (Reference) & \\
\hline & Pharmacist & $16 / 84$ & $(19.0)$ & $1.329(0.696-2.539)$ & 0.389 & $1.287(0.630-2.629)$ & 0.488 \\
\hline & Medical doctor & $47 / 170$ & $(27.6)$ & $2.158(1.329-3.505)$ & 0.002 & $2.003(0.932-4.305)$ & 0.075 \\
\hline \multirow{2}{*}{$\begin{array}{l}\text { Participation in } \\
\text { clinical trial }\end{array}$} & No & $61 / 352$ & (17.3) & 1 (Reference) & & 1 (Reference) & \\
\hline & Yes & $39 / 148$ & (26.4) & $1.707(1.079-2.699)$ & 0.022 & $1.139(0.628-2.063)$ & 0.668 \\
\hline
\end{tabular}

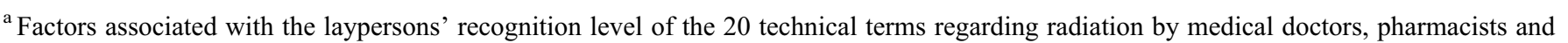
nurses were analyzed by binary logistic regression model to assess the crude odds ratio (OR) and adjusted OR. The score was summed, and 61 was adopted as a cut-off index, indicating that a total score $\geq 61$ for recognition level of the 20 technical terms regarding radiation meant that "laypersons know".

${ }^{\mathrm{b}}$ Crude odds ratio and $95 \%$ confidence interval.

${ }^{\mathrm{c}}$ Adjusted odds ratio for sex, age, occupation, participation of clinical trial and $95 \%$ confidence interval.

$\mathrm{N}$ : number of subjects.

meeting of risk communication (58.3\%), - public relations by the local government $(36.9 \%)$, and radio again ranked the lowest $(16.7 \%) .51 .2 \%$ of nurses considered TV to be a resource, followed by the internet $(46.7 \%)$, newspaper (41.7\%), and again radio ranked the lowest (26.8\%). 53.3\% of nurses believed that academic journals/technical books were reliable, followed by forum/meeting of risk communication (51.6\%), public relations by central government $(39.4 \%)$, and finally radio $(21.1 \%) .57 .5 \%$ of laypersons considered TV to be a resource, followed by the internet $(53.6 \%)$, newspaper $(52.8 \%)$, with radio ranking lowest $(25.9 \%) .51 .2 \%$ of laypersons believed that academic journals/technical books were reliable, followed by forum/meeting of risk communication $(42.0 \%)$, newspaper $(41.3 \%)$, and finally radio $(26.2 \%)$.

In terms of factors associated with the laypersons' expected recognition level of the 20 technical terms regarding radiation by medical doctors, pharmacists and nurses, with the unadjusted analysis, there was a statistically significant association with the expectation that "laypersons know" as shown in Table 6 . When analyzing the details of answers by medical staff with adjusted odds ratio, no significant difference was evidenced according to socio-demographic parameters such as age, sex, occupation or participation in clinical trials.

\section{Discussion}

First of all, as part of risk communication after the great East Japan Earthquake of 2011, healthcare workers in Japan were considered to be advocates who provided information regarding the effect of radiation on the human body. This study showed that in the actual fields of risk communication, medical doctors were more apt than pharmacists and nurses to estimate that laypersons knew technical terms regarding radiation. In contrast, our previous research demonstrated that the laypersons' expected level of recognition of medical terms, not of radiation, was higher when estimated by nurses, followed by that when estimated by pharmacists and medical doctors (Yoshida et al., 2017). Advocates, such as medical doctors, should consider the results of this study, and try to deliver information on radiation using simple explanations in forumstyle risk communication programs.

Regarding the knowledge that should be delivered at a forum-style risk-communication program, the item of most interest for laypersons was the "risk of radiation effects", followed by "effectiveness of food standards as a radiation countermeasure", while the uncertainty of the effects of radiation was of least interest among the 10 items. It seemed that since uncertainty regarding information caused anxiety, that information was the least interesting for laypersons. In contrast, for medical doctors, the item perceived to be of most interest to the public was "scientific facts", followed by "risk of radiation effects", and "setting the basis for the standards". For pharmacists, the item perceived to be of most interest was "scientific facts", followed by "Radiation effects on children". For nurses, the item perceived to be of most interest was "risk of radiation effects", followed by "radiation effects on children". These results showed that there were the differences between public perception of risks and the belief by the medical staff of what the public risk perception was. In order to enhance public understanding, medical doctors believed that this kind of information should be delivered carefully. In contrast, the effectiveness of the food standards as countermeasures had the fifth highest interest level for laypersons; 
however, in these results medical doctors ranked food-based measures at the seventh highest interest level. A previous study demonstrated that food-based measures were strongly related to absorption by the body, so that a facilitator was required to consider that their explanations are more scientifically sound and plain in order to avoid misconceptions and depression (Ohtsuru et al., 2015). Lochard et al. also mentioned that the importance of the dialogue meetings initiated by ICRP at Fukushima and that these dialogues were centered on the public interest and not on what the medical staff belief about the public interest. (Lochard et al., 2019). Therefore, healthcare workers, especially medical doctors, should consider this difference when conducting risk communication with laypersons.

The most popular source was academic journals/technical books, followed by the internet, and forum or meeting of risk communication. In addition, academic journals/technical books, and forum or meeting of risk communication scored the highest in reliability as sources of information. Medical doctors had greater access to academic journal/technical books, and forums/meetings of risk communication owing to their profession and were required to explain the appropriate information to laypersons that also relied on them. Our previous research demonstrated that the forum/meeting of risk communication was accepted as a reliable source (Yoshida et al., 2017), therefore, this activity should be continued rigorously and vigorously. From the viewpoint of the laypersons, public relations conducted by the local and central governments occupied the fourth and fifth positions, respectively, as sources of information. One of the reasons was that, to some extent, the attitude of the government seemed apt to be overbearing to laypersons. Therefore, medical doctors were expected to be intermediaries on behalf of the local and central government officers in communications with laypersons.

About one-fifth of each respondent group agreed that "by performing the risk avoidance behavior according to the criteria defined by the government, laypersons can sufficiently avoid health effects". Medical doctors also relied less on governments in this aspect. In contrast, there was a statistically significant difference in the risk avoidance behavior regarding radiation. In terms of "taking into account the uncertainty of the risk assessment, laypersons should avoid the risk", the highest values of percentage $(54.0 \%)$ were marked by laypersons. From the viewpoint of personal responsibility to avoid risk, there was clearly mistrust of governments by the public. In terms of this issue, healthcare workers trusted the central government. Under the universal health insurance system of Japan, the activities of healthcare workers are evaluated when medical fees were set by the central government every two years, and they are paid by public agencies, so they relied more on the central government than laypersons do. A previous study demonstrated that trust was one of the most important factors in risk communication that made it more effective and smooth (Jensen, 2004); therefore, from the viewpoint of trust toward the lecturers and facilitators, healthcare workers have also had good and suitable positions when compared to central/local government staffs.

The results of this study also showed that even healthcare workers who were experts in the field of healthcare did not have complete information about the concepts of ALARA and
Regulatory Science. Although the concepts of ALARA and Regulatory Science were crucial for the success of risk communication among various stakeholders, the percentage of medical doctors who had correct perception of the concept of ALARA and Regulatory Sciences was $23.5 \%$ and $16.5 \%$, respectively. In order to improve the knowledge of healthcare workers in Japan regarding these two concepts, the concept of ALARA and Regulatory Sciences should be added in their education curriculum. We have been conducting a similar study in Japan with the viewpoint of obtaining the percentage of academic experts who have correct perception of the concept of ALARA and Regulatory Sciences.

Compared to internet surveys, an interview survey usually obtains more concise and precise information about the degree of the recognition level of technical terms. Recently, internet surveys have been accepted in the field of medical sociology in Japan. However, we considered this a limitation of this study.

In conclusion, to reduce the anxiety and/or stress caused by radiation exposure in the Tohoku region, risk communication about radiation exposure to the human body should be conducted continuously, vigorously, and rigorously by healthcare workers as lecturers.

\section{Acknowledgments}

This study was partially supported by JSPS KAKENHI Grant Number 16K09130 from the Japanese Society for the Promotion of Science.

Conflicts of interest. The authors declare that they have no conflicts of interest.

\section{References}

Bromet EJ. 2014. Emotional consequences of nuclear power plant disasters. Health Phys 106: 206-210.

Coates R, Czarwinski R. 2018. IRPA Consultation: is the system of protection "fit for purpose" and can it be readily communicated? Views of the radiation protection professionals. J Radiol Prot 38: 440-455.

Goto A, Bromet EJ, Fujimori K. 2015. Immediate effects of the Fukushima nuclear power plant disaster on depressive symptoms among mothers with infants: A prefectural-wide cross-sectional study from the Fukushima Health Management Survey. BMC Psychiatry 15: 59.

Hayashi Y. 2009. Scientific basis for risk analysis of food-related substances with particular reference to health effects on children. $J$ Toxicol Sci 34 (Suppl 2): SP201-7.

International Commission on Radiological Protection (ICRP) Publication 103 (2007).

Jensen KK. 2004. BSE in the UK: Why the risk communication strategy failed. J Agric Environ Ethics 17: 405-423.

Kuroda Y, Iwasa H, Orui M, Moriyama N, Nakayama C, Yasumura S. 2018. Association between health literacy and radiation anxiety among residents after a nuclear accident: Comparison between evacuated and non-evacuated areas. Int J Environ Res Public Health 15(7).

Lochard J, Schneider T, Ando R, Niwa O, Clement C, Lecomte JF, Tada JI. 2019. An overview of the dialogue meetings initiated by ICRP in Japan after the Fukushima accident. Radioprotection 54 (2): $87-101$. 
Nakashima K, et al. 2015. Radiocesium concentrations in wild mushrooms collected in Kawauchi Village after the accident at the Fukushima Daiichi Nuclear Power Plant. PEERJ 3: e1427. https://doi.org/10.7717/peerj.1427.

Ohtsuru A, et al. 2015. Nuclear disasters and health: lessons learned, challenges, and proposals. Lancet 386: 489-497.

Poston JW Sr., Fortieth Lauriston S. 2017. Taylor Lecture: Radiation Protection and Regulatory Science. Health Phys 112(2): 193-198

Sekizawa J. 2013. Other aspects of BSE issues in East Asian countries. Risk Anal 33: 1952-1957.

Staudenherz A, Leitha T. 2011. Medical preparedness in radiation accidents: a matter of logistics and communication not treatment! Int J Occup Environ Med 2: 133-142.

Tsuchida S. 2018. Psychology of safety and risk: the form of safety created by the mind (in Japanese). Baifukan 89-119.

amaguchi T. 2013. Social imaginary and dilemmas of policy practice: The food safety arena in Japan. Food Policy 45: 167-173.
Yoshida Y, Yoshida Y. 2014. Patient's recognition level of medical terms as estimated by pharmacists. Environ Health Prev Med 19: 414-421.

Yoshida Y, Yoshida Y. 2015. Patients' level of medical term recognition as estimated by healthcare workers. Nagoya J Med Sci 77: $123-132$.

Yoshida Y, Yoshida Y, Motoyoshi T, Saito M, Saito MA, Hayase T. 2013. Study of perception gaps in pharmaceutical terms and related issues between laypeople and medical practitioners (in Japanese). Nihon Eiseigaku Zasshi Japanese Journal of Hygiene 68: 126-137.

Yoshida Y, et al. 2017. Level of perception of technical terms regarding the effect of radiation on the human body by residents of Japan. Environ Health Prev Med 22: 73.

Yumiya Y, Goto A, Murakami M, Ohira T, Rudd RE. 2019. Communication between health professionals and community residents in Fukushima: A focus on the feedback loop. Health Commun 1-9.

Cite this article as: Yoshida Y, Yoshida Y. 2020. Medical staff perceptions of risk communication needs for the public and comparison with the needs expressed by the public. Radioprotection 55(3): 199-206 